\title{
From 2D to 3D: Critical Casimir interactions and phase behavior of colloidal hard spheres in a near-critical solvent
}

Nikos Tasios and Marjolein Dijkstra

Citation: The Journal of Chemical Physics 146, 134903 (2017); doi: 10.1063/1.4979518

View online: http://dx.doi.org/10.1063/1.4979518

View Table of Contents: http://aip.scitation.org/toc/jcp/146/13

Published by the American Institute of Physics

\section{Articles you may be interested in}

A Gaussian theory for fluctuations in simple liquids

The Journal of Chemical Physics 146, 134507134507 (2017); 10.1063/1.4979659

A simulation study on the phase behavior of hard rhombic platelets

The Journal of Chemical Physics 146, 144901144901 (2017); 10.1063/1.4979517

Colloid-polymer mixtures under slit confinement

The Journal of Chemical Physics 146, 104903104903 (2017); 10.1063/1.4977831

Phase behaviour of quasicrystal forming systems of core-corona particles

The Journal of Chemical Physics 146, 114901114901 (2017); 10.1063/1.4977934

Surface tension of a Yukawa fluid according to mean-field theory

The Journal of Chemical Physics 146, 134701134701 (2017); 10.1063/1.4979203

Thermodynamics of two-dimensional Yukawa systems across coupling regimes

The Journal of Chemical Physics 146, 134702134702 (2017); 10.1063/1.4979325

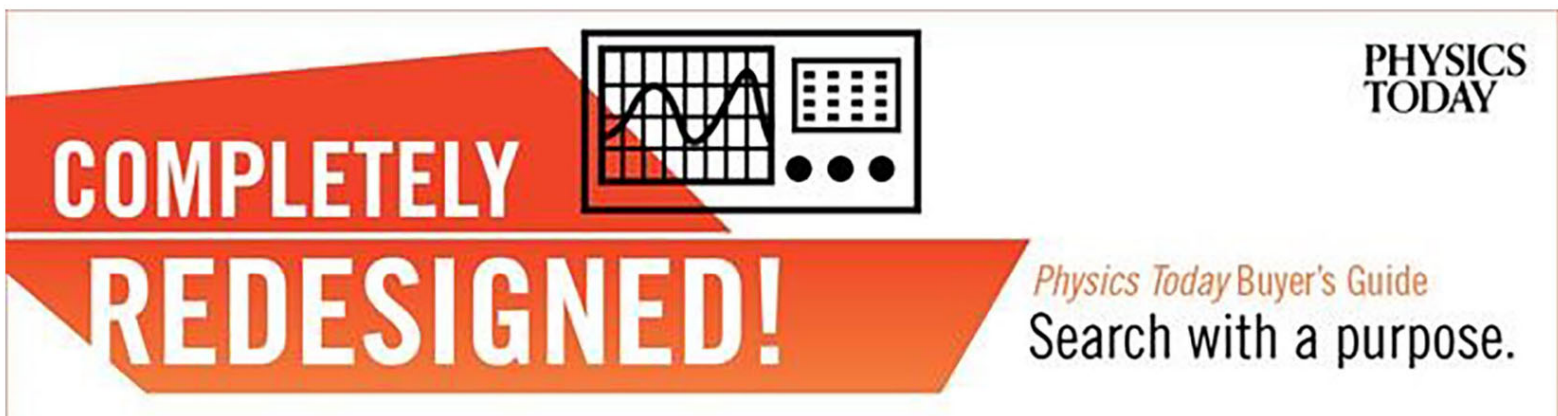




\title{
From 2D to 3D: Critical Casimir interactions and phase behavior of colloidal hard spheres in a near-critical solvent
}

\author{
Nikos Tasios and Marjolein Dijkstra \\ Soft Condensed Matter, Debye Institute for Nanomaterials Science, Utrecht University, Princetonplein 1, \\ 3584 CC Utrecht, The Netherlands
}

(Received 7 December 2016; accepted 7 March 2017; published online 7 April 2017)

\begin{abstract}
Colloids dispersed in a binary solvent mixture experience long-ranged solvent-mediated interactions (critical Casimir forces) upon approaching the critical demixing point of the solvent mixture. The range of the interaction is set by the bulk correlation length of the solvent mixture, which diverges upon approaching the critical point. This presents a great opportunity to realize the reversible selfassembly of colloids by tuning the proximity to the critical point of the solvent. Here, we develop a rejection-free geometric cluster algorithm to study the full ternary mixture of colloidal hard spheres suspended in an explicit three-dimensional lattice model for the solvent mixture using extensive Monte Carlo simulations. The phase diagram displays stable colloidal gas, liquid, and crystal phases, as well as broad gas-liquid and gas-crystal phase coexistence, and pronounced fractionation of the solvent in the coexisting colloid phases. The topology of the phase diagram in our three-dimensional study shows striking resemblance to that of our previous studies carried out in two dimensions. Published by AIP Publishing. [http://dx.doi.org/10.1063/1.4979518]
\end{abstract}

\section{INTRODUCTION}

Upon approaching the critical "demixing" point of a binary solvent mixture, the local composition of the mixture fluctuates with a bulk correlation length that diverges as $\xi \sim\left|T-T_{C}\right|^{-v}$, where $\xi$ is the correlation length, $T$ is the temperature, $T_{C}$ is the critical temperature, and $v$ is the "critical exponent." Fisher and de Gennes showed in $1978,{ }^{1}$ in a very important theoretical study, that two plates immersed in a binary solvent mixture at a separation $L \sim \xi$ and preferentially adsorbing one of the solvent species feel a long-ranged solvent-mediated (SM) interaction due to the critical fluctuations. At the critical point, the SM interaction between the plates is "universal" (it does not depend on the microscopic details, rather on the adsorption preference and the dimension of the system) and diverges algebraically. For plates with a preference for the same solvent species, the SM interaction is attractive, and in the case that the plates preferentially adsorb different solvent species, the SM interaction is repulsive. This type of fluctuation-induced interaction is not unique to binary solvent mixtures, but manifests itself for any fluid in the proximity of its critical point, and has come to be known as "critical Casimir" interaction. ${ }^{2-4}$ The critical Casimir interaction owes its name to the similarities that it is believed to share with the quantum-mechanical Casimir ${ }^{5}$ effect which arises between two conducting plates as a result of the confinement of the vacuum fluctuations of the electromagnetic field.

The critical Casimir interaction has been thoroughly studied, especially in the context of solvent mixtures. For instance, the critical Casimir interaction between two static planar walls has been the subject of numerous theoretical studies ${ }^{2-4,6,7}$ and has been extended to a pair of spherical particles using the Derjaguin approximation ${ }^{8}$ which approximates the spherical surface with a number of planar walls. Experimentally, the critical Casimir force between polystyrene particles and a chemically treated silica wall, in a near-critical waterlutidine mixture, was measured using total internal reflection microscopy in $2008^{9}$ and showed quantitative agreement with theoretical predictions. As the critical Casimir interactions depend directly on the bulk correlation length, these are very sensitive to the temperature and composition of the mixture, and this raises prospects to tune the self-assembly process of, e.g., suspended colloids, reversibly and in an in situ fashion. In this context, Beysens and Estève studied already in 1985 the thickness of the adsorbed films at the surface of silica spheres immersed in a binary liquid mixture as a function of temperature and solvent composition, and they determined the locus of points in the temperaturesolvent composition plane where colloidal spheres aggregate reversibly. ${ }^{10}$ In 2008, charge-stabilized polystyrene particles in a binary solvent mixture were studied using x-ray scattering, and reversible phase transitions between gas and solid, and gas and liquid phases were observed. ${ }^{11}$ Subsequently, colloidal aggregation in near-critical solvents was studied in real space using confocal microscopy. Aggregation was observed in a remarkably wide temperature range of as much as $15^{\circ} \mathrm{C} .{ }^{12}$ More recently, colloidal phase transitions were studied as a function of temperature using confocal microscopy. ${ }^{13}$

Theoretical and simulation studies on the phase behavior and structure of the full ternary mixture of colloidal particles moving around in an explicit solvent mixture are hampered by the huge differences in length and time scales between the colloids, solvent species, and the diverging bulk correlation length of the solvent mixture. As such, in most theoretical and simulation approaches, such as the works of Mohry et al. ${ }^{14-16}$ Nguyen et al., ${ }^{13,17}$ and Dang et al.,${ }^{18}$ the binary solvent mixture is taken to be a structureless implicit background. This approach fails to capture important elements such as 
the fractionation of the solvent in the coexisting colloidal phases. In a series of recent studies, ${ }^{19-21}$ we were able to capture the above-mentioned important elements by using a simple lattice-gas binary solvent model with embedded colloids interacting through first neighbor and excluded volume interactions with the solvent. The model, although simple, contains all the necessary physics to describe the SM interactions between colloids. Since an explicit solvent is used, fractionation and many-body effects arise naturally. Due to the large difference in length scales between the colloid and solvent, and the critical slowing down as the ternary mixture critical point is approached, studying a three-dimensional system using Monte Carlo simulations was deemed computationally unfeasible, and in Refs. 19 and 21 we presented results for a two-dimensional system only. We suggested that the overall topology of the phase diagram should be similar in three dimensions.

In the current work, we study the phase behavior of preferentially adsorbing colloidal spheres embedded in a binary solvent mixture in three dimensions, using Monte Carlo simulations. We use the same model, generalized to $3 \mathrm{D}$, as in Refs. 19 and 21, which we describe in Section II A. The study was made possible by the development of a cluster algorithm which we describe in Section II B. Cluster algorithms are known to alleviate problems of critical slowing down and of low acceptance when performing Monte Carlo moves on constituents of very different sizes. ${ }^{22-24}$ We mention that a geometric cluster algorithm was also developed recently by Hobrecht and Hucht ${ }^{25}$ for a similar lattice model. We focus on the supercritical, $\tau>0$, region of the pure solvent mixture reservoir, where we note that the lattice-gas model has an upper critical solution temperature. Here, $\tau=\left(T-T_{C}\right) / T_{C}$ is the reduced temperature of the system-it is important to recognize that $T_{C}$, here, refers to the critical temperature of the solvent in the absence of colloids. The motivation behind this choice is to avoid the added complexity of having to deal with subcritical effects such as capillary bridging. Of course, the model can be used in any scenario.

This communication is organized as follows. In Section II, we introduce our model of a ternary solvent-solvent-colloid mixture and describe the cluster algorithm which made this study possible. In Section III, we present the phase diagram in the solvent chemical potential difference, $\Delta \mu_{S}$, and colloid packing fraction, $\eta$, representations, for varying temperatures. In Section IV, we make some conclusions.

\section{MODEL AND METHODS}

\section{A. Model}

The underlying incompressible binary $A B$ solvent in our system is described by a simple lattice-gas model, which is completely isomorphic to the Ising model. To drive $A B$ demixing, an energy penalty $\epsilon / 2>0$ is assigned to every nearestneighbor $A B$ pair. Colloids $C$ of radius $R$ are embedded on center-of-mass positions $\boldsymbol{p}$, as a collection of lattice sites $\boldsymbol{n}$, for which $|\boldsymbol{n}-\boldsymbol{p}|^{2} \leq R^{2}$. Additionally, excluded volume rules are imposed on each lattice site, and an energy gain of $-\alpha \epsilon / 2$, with $\alpha \geq 0$, is introduced for each nearest-neighbor $B C$ pair, to mimic preferential adsorption of species $B$ onto the colloids,
$C$. The Hamiltonian of the system is thus given by

$$
\begin{aligned}
H= & H_{C}+\frac{\epsilon}{4} \sum_{\langle i, j\rangle}\left(1-s_{i} s_{j}\right)\left(1-n_{i}\right)\left(1-n_{j}\right) \\
& -\frac{\alpha \epsilon}{4} \sum_{\langle i, j\rangle} n_{i}\left(1-n_{j}\right)\left(1+s_{j}\right),
\end{aligned}
$$

where $s_{i}=-1$, if site $i$ is occupied by species $A, s_{i}=1$, if site $i$ is occupied by species $B$, and $s_{i}=0$ otherwise. $n_{i}$ is the colloid occupancy which is equal to 1 if site $i$ is occupied by a colloid $C$, and 0 otherwise. The Hamiltonian $H_{C}$ is infinite if any pair of colloids overlaps, otherwise it is zero.

When $\alpha=0$, colloids have no preference for either of the two solvent species, and we call these neutrally adsorbing colloids. The $A B$ solvent mixture, in the absence of colloids, has a critical temperature given by $k_{B} T_{C} / \epsilon \approx 1.12788$. $^{26}$

The ternary $A B$-solvent-colloid mixture is studied using Monte Carlo simulations. In our implementation, a Monte Carlo cycle consists of the following moves:

- $N_{s}$ attempts to flip the spin variable $s_{i}$ at a random lattice site $i$. Note that we reject the move if $n_{i}=1$, i.e., if the random site, $i$, is occupied by a colloid.

- $N_{C}$ attempts to translate a random colloid by one lattice spacing.

- A cluster move.

Here $N_{s}=N_{A}+N_{B}$ denotes the number of solvent sites with $N_{A}$ the number of $A$ species and $N_{B}$ the number of $B$ species sites. $N_{C}$ refers to the number of colloids. Solvent moves are accepted using the standard Metropolis criterion,

$$
a\left(N_{B} \rightarrow N_{B} \pm 1\right)=\min \left[1, e^{-\beta\left(\Delta H \mp \varepsilon \Delta \mu_{s}\right)}\right],
$$

where $\Delta \mu_{s}=\left(\mu_{B}-\mu_{A}\right) / \epsilon$ is the reduced chemical potential difference of the solvent and $\Delta H$ is the difference in potential energy as given by Equation (1). Colloidal particle translations are performed by choosing a random direction, $\pm \hat{x}, \pm \hat{y}$, or $\pm \hat{z}$ and point reflecting sites for which $n_{i}$ has changed after the translation. The move is again accepted using the Metropolis criterion, $a=\min \left(1, e^{-\beta \Delta H}\right)$. For a more detailed description, see our previous publication. ${ }^{21}$

\section{B. Cluster algorithm}

The model we presented above involves species of significantly different sizes, known to cause difficulties when performing Monte Carlo moves, such as translations. ${ }^{24,27,28}$ This, coupled with the fact that solvent species $B$ can adsorb strongly to the colloid surface and that critical slowing-down upon approaching the ternary mixture critical point is encountered, leads to extremely low acceptance rates, and exponentially longer equilibration times as a function of temperature. ${ }^{29}$ It should be noted that in the two-dimensional model of Refs. 19 and 21, colloid translations have a much higher acceptance rate than in our current three-dimensional model; colloids in 2D have significantly fewer surface sites with which they can interact with solvent species, than colloids of the same radius in $3 \mathrm{D}$. This makes displacements of colloids much more difficult in higher dimensions and with increasing colloid radius. 
To overcome the low acceptance of colloid translations and the critical slowing down, we developed a geometric cluster algorithm based on the works by Heringa and Blöte ${ }^{22,23}$ and Ashton et al. ${ }^{24}$ Geometric cluster algorithms employ a symmetry operation in order to transform a cluster. Provided this symmetry operation is a global symmetry of the Hamiltonian, the cluster algorithm can be shown to obey a detailed balance. ${ }^{23}$ Our algorithm exploits the fact that the Hamiltonian is symmetric under the reflection of the lattice about an arbitrary pivot point. In the implementation of our model, a lattice site at index $i$ is represented by the variable $t_{i}$ which takes the values $t_{i} \in\left[0, N_{C}+2\right]$ corresponding to the species that occupies lattice site $i$, with $A \equiv 0, B \equiv 1$, and colloid $n, C_{n} \equiv n+2$. It is also easy to see that $s_{i}=t_{i}-1, n_{i}=0$ when $t_{i}<2$, and $n_{i}=1$ when $t_{i} \geq 2$. In the same spirit as Ref. 22, we use primed identifiers, i.e., $i^{\prime}$, to denote identifiers that are mapped from the original by a symmetry transformation, in this case a point reflection about a random pivot point. As is the case with most cluster algorithm implementations, since the algorithm is rejection free, we can point-reflect lattice sites as they are added to the cluster instead of reflecting the whole cluster at the end of the algorithm. To this end, we keep track of the lattice sites that are added to the cluster by marking them as added to the cluster. The geometric cluster algorithm, modified for our model, consists of the following steps:

1. Choose a random colloid and lattice pivot point, $r$, about which point reflection is to be performed. The site at the center of the colloid is denoted as $i$, and the site reflected about the pivot point $r, i^{\prime}$. Figure 1 presents a schematic picture.

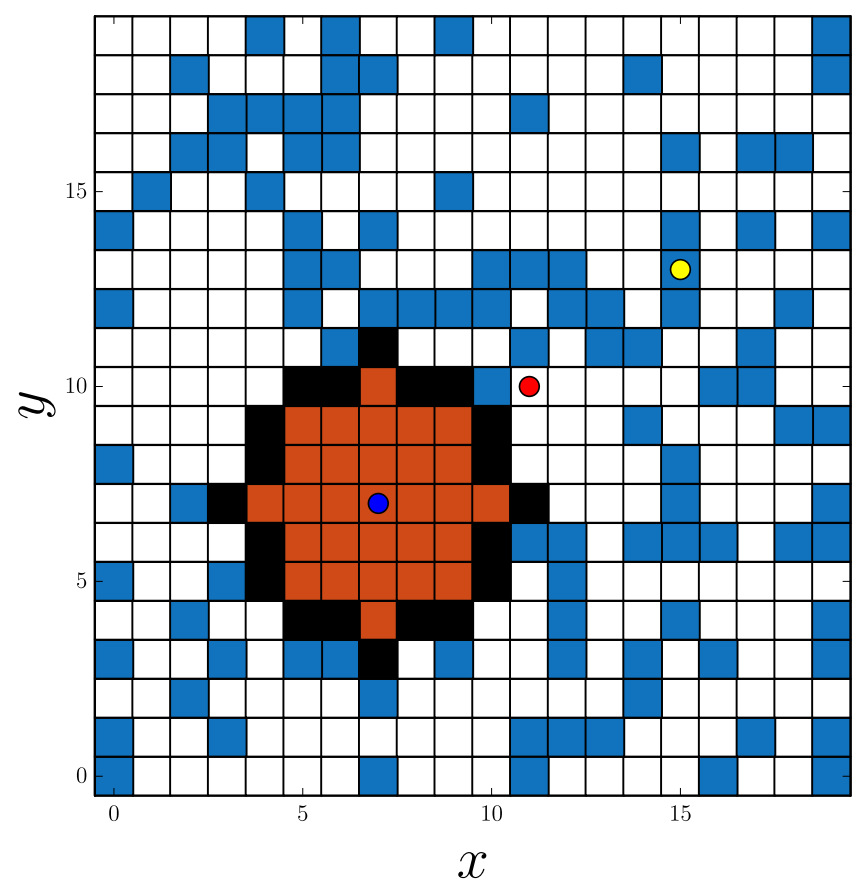

FIG. 1. When performing a cluster move, a random colloid and a random pivot point, $r$ (red circle at $(11,10)$ ), are chosen. Starting with its central site at lattice index $i$ (blue circle at $(7,7)$ ), and the site reflected about the pivot point, $i^{\prime}$ (yellow circle at $(15,13)$ ), neighboring sites are added to the cluster according to the cluster move rules described in the text.
2. Interchange the species variables $t_{i}$ and $t_{i^{\prime}}$ of sites $i$ and $i^{\prime}$ and mark them as added to the cluster.

3. For all neighboring sites, $k$ and $k^{\prime}$, of site $i$ and corresponding reflected site $i^{\prime}$, that have not been added to the cluster, do the following:

3.1. If $t_{i} \geq 2$, i.e., it belongs to a colloid, and $t_{k^{\prime}}=t_{i}$, i.e., sites $i$ and $k^{\prime}$ belong to the same colloid, or if site $t_{i^{\prime}} \geq 2$ and site $t_{k}=t_{i^{\prime}}$, interchange the species variables $t_{k}$ and $t_{k^{\prime}}$ and mark sites $k$ and $k^{\prime}$ as added to the cluster.

3.2. Else, calculate the energy difference, $\Delta H$, associated with interchanging $t_{k}$ and $t_{k^{\prime}}$. If $\Delta H>0$, interchange the species variables $t_{k}$ and $t_{k^{\prime}}$ and mark sites $k$ and $k^{\prime}$ as added to the cluster with probability $1-e^{-\beta \Delta H}$.

4. For each site that was added to the cluster in step 3 , denote these as $i$, and repeat steps 3 and 4 , or terminate if no sites were added.

Step 3.1 is the equivalent of saying that nearest-neighbor pairs belonging to the same colloid are bonded infinitely strongly, such that $\Delta H=\infty$. This ensures that entire colloids are added to the cluster if at least one of their sites is added. The geometric cluster algorithm described above significantly reduces the relaxation time of the system and renders translations rejection free. When a cluster becomes percolating, the cluster move may leave the system unchanged. For this reason, we also attempt standard colloid translations. In our implementation, we found it to be more effective to restrict the random pivot point to a small window centred around the colloid.

\section{RESULTS}

The focus of this communication is to show that largescale simulations of a ternary $A B$-solvent-colloid mixture in $3 \mathrm{D}$ and in near-critical solvent conditions are possible using a cluster algorithm. We do this by assessing the phase behavior and compare the overall topology of the resulting 3D phase diagram with that of a two-dimensional system. ${ }^{19,21}$ This comparison is achieved by calculating the solvent chemical potential difference, $\Delta \mu_{s}$, vs colloid packing fraction, $\eta$, phase diagrams of the ternary mixture at different reduced temperatures, $\tau$.

To calculate the phase diagrams, we employ the direct coexistence method ${ }^{21}$ in which an elongated box is used to facilitate the formation of an interface between two phases. This allows us to calculate density profiles along the direction normal to the interface, from which the colloid coexistence packing fractions $\eta$ can be "read off" for a fixed solvent chemical potential, $\Delta \mu_{s}$. We thus treat the colloids in the canonical ensemble, whereas the solvent is treated grandcanonically; see Ref. 21. Throughout this work, a simulation box with dimensions of $128 \times 128 \times 256$ lattice sites is used; this is large enough to avoid significant finite-size effects.

By calculating the density profiles at different chemical potentials, $\Delta \mu_{s}$, we determined the phase diagrams for three different temperatures, $\tau=0.025,0.05$, and 0.075 , as shown in Figure 2, and for a colloid radius $R=6$, and a fixed value of the adsorption strength used previously in 2D in Refs. 19 and 


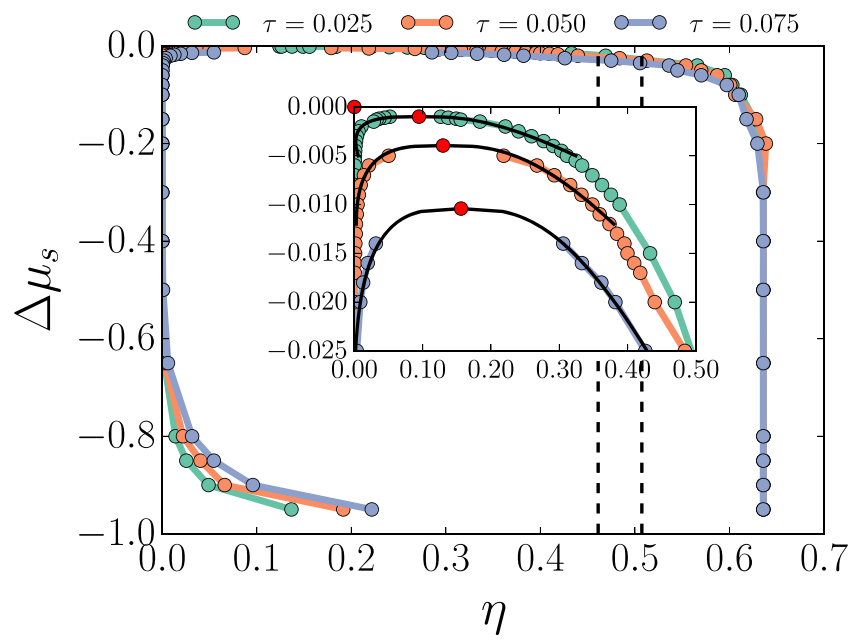

FIG. 2. Phase diagram of the $A B C$ ternary mixture, computed using the direct coexistence method, for three fixed temperatures $\tau=0.025$ (green), $\tau=0.05$ (orange), and $\tau=0.075$ (blue) with colloid radius $R=6$ and colloid-solvent adsorption strength $\alpha=0$.6. In this solvent chemical potential, $\Delta \mu_{s}$, vs colloid packing fraction, $\eta$, representation, the tie lines are horizontal. The red circles and black lines in the inset are the critical points and binodals, respectively, obtained by least-squares fitting of Equation (3). Note that the vertical dashed lines denote fluid-solid coexistence for our lattice representation of the pure hard-sphere system; see text.

21, $\alpha=0.6$-we focus on the supercritical, $B$-rich, $\Delta \mu_{s}<0$ region, where capillary effects are absent. We observe colloidal gas (G), liquid (L), and crystal (X) phases, as well as phase coexistence between colloidal gas and liquid phases, and colloidal gas and crystal phases, with pronounced fractionation of the solvent. In Figure 3 we show examples of G-L (b) and G-X (c) coexistence, along with a supercritical configuration (a). In the inset of the phase diagram in Figure 2, we present a close-up of coexistence in the near critical region. We also plot estimates of the critical points, obtained by a least-squares fit to the equation ${ }^{30-33}$

$$
\eta_{ \pm}-\eta_{c}=A\left|\Delta \mu_{s}-\Delta \mu_{s}^{c}\right| \pm \frac{1}{2} B\left|\Delta \mu_{s}-\Delta \mu_{s}^{c}\right|^{\beta},
$$

where $\eta_{ \pm}$stands for the coexisting colloidal liquid/gas packing fraction, with $\eta_{c}$ its critical value, and $A, B$, are fit parameters. The critical exponent, $\beta$, was fixed to its value for the three-dimensional Ising universality class, $\beta=0.3258 .{ }^{34}$ Note that we have substituted temperature with the solvent chemical potential, $\Delta \mu_{s}$, with $\Delta \mu_{s}^{c}$ being the solvent chemical potential at the critical point of the ternary mixture. We note that we use Equation (3) as a fit to our result, only in order to guide the eye. From the phase diagram in Figure 2, we observe broad G-X coexistence for $\Delta \mu_{s}<-0.1$, and a $\mathrm{L}-\mathrm{X}$ coexistence region for $-0.1<\Delta \mu_{s}<-0.9$, which becomes narrower upon decreasing $\Delta \mu_{s}$. In addition, we expect to recover the fluid-crystal transition of pure hard spheres at $\Delta \mu_{s}= \pm \infty$, corresponding to hard spheres in either a pure $A$ or a pure $B$ solvent background. We thus find that the G-L binodal ends in a G-L-X triple point at lower $\Delta \mu_{s}$. Assuming that the colloidal spheres are sufficiently large and that lattice effects are minimal, the system of pure hard spheres should exhibit fluid-crystal coexistence at packing fractions $\eta=0.4915$ and $\eta=0.5428 .{ }^{35}$ From our lattice simulations, we find that the actual coexistence packing fractions are $\eta=0.4611$ and $\eta=0.5073$, drawn as dashed lines in Figure 2. This should come as no surprise; the spheres (a)

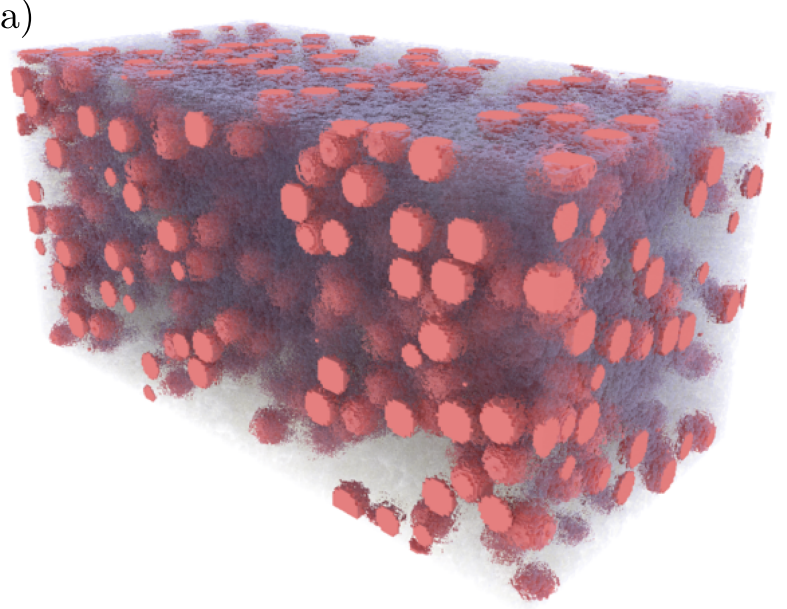

(b)

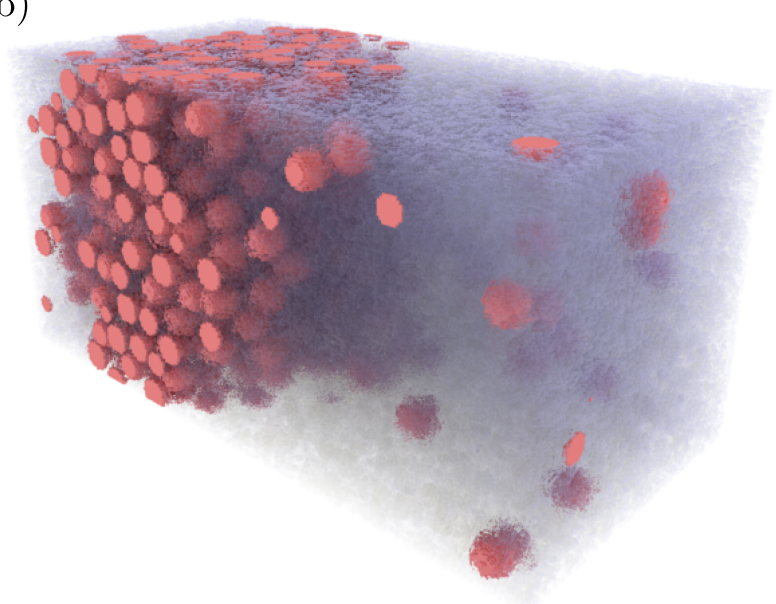

(c)

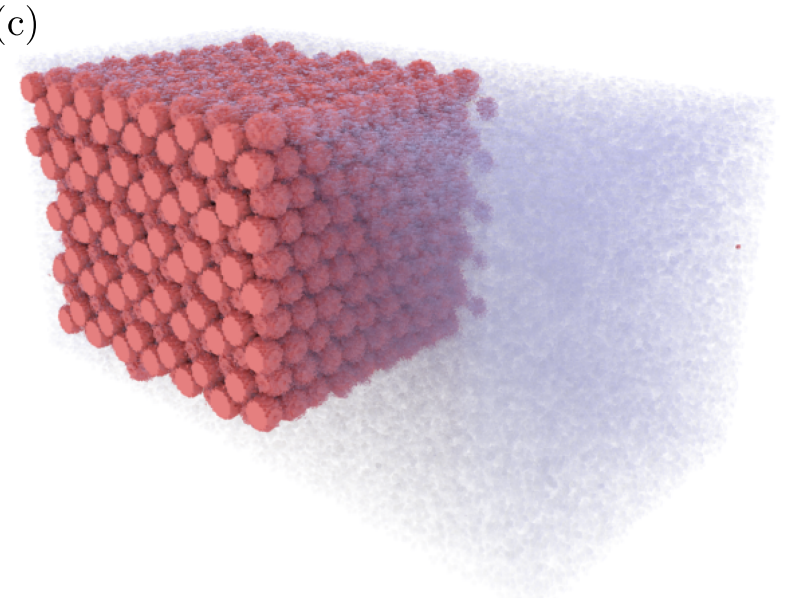

FIG. 3. Representative configurations of our ternary colloid-solvent mixture model at reduced temperature $\tau=0.05$, colloid radius $R=6$, and for different chemical potentials, (a) $\Delta \mu_{s}=-0.002$ (supercritical), (b) $\Delta \mu_{s}=-0.008$ (G-L coexistence), and (c) $\Delta \mu_{s}=-0.3$ (G-X coexistence). The pink-colored particles represent the colloids, while the grey-colored ones, the solvent $B$ species. The configurations were visualized using a path tracing renderer.

in our model are rather small such that the lattice discretization affects their packing and hence the coexistence packing fractions.

From the phase diagram in Figure 2 it is clear that the topology is similar to that of the two-dimensional system. ${ }^{19,21}$ The coexistence curves of the three-dimensional system are 
broader, mainly due to the fact that the particle surface is proportional to $R^{2}$ as opposed to $R$ in 2D; the colloidal particles in $3 \mathrm{D}$ are overall adsorbing more strongly for the same value of $\alpha$. The phase diagrams also exhibit the same trends when decreasing the temperature, $\tau$; the two-phase G-L(X) coexistence region broadens, and the critical point of the ternary system shifts towards the critical point of the pure solvent reservoir.

\section{CONCLUSION}

Computer simulations of a suspension of colloidal particles in a near critical solvent mixture in three dimensions were previously prohibited due to slow equilibration arising from large differences in length and time scales between the individual components, the strong adsorption of the solvent on the colloids, and the critical slowing down. In this work, we circumvented these problems by developing a rejection-free geometric cluster algorithm which takes advantage of the point reflection symmetry of the Hamiltonian of our lattice-based model.

Using the direct coexistence method, we determined the phase behavior of colloidal hard spheres in a near-critical solvent mixture. We determined the gas-liquid and gas-solid binodals for three different supercritical reduced temperatures, $\tau=0.025,0.05$, and 0.075 , close to the solvent reservoir's critical point. We found the same qualitative features as in the two-dimensional case: (i) we observe that the upper gasliquid critical point of the ternary colloid-solvent mixture shifts towards lower solvent chemical potential differences $\Delta \mu_{S}$ and higher colloid packing fractions, $\eta$, upon adding colloids, and (ii) we find broad gas-liquid phase and gas-solid phase coexistence, with a significant fractionation of the solvent species favored by the colloids.

In conclusion, we have shown that our cluster algorithm allows us to simulate efficiently the full ternary colloid-solvent mixture in 3D. Moreover we also demonstrated that the key features of our previous results on the phase behavior and structure in two dimensions are present in three dimensions.

\section{ACKNOWLEDGMENTS}

We acknowledge financial support from an NWO-Echo grand and an NWO-EW grant for computing time on the Dutch supercomputer Cartesius. We thank J. R. Edison, R. Evans, A. Macioełek, and D. Ashton for many stimulating and inspiring discussions.
${ }^{1}$ M. E. Fisher, P.-G. de Gennes, and C. R. Hebd, Seances Acad. Sci. 287, 207 (1978).

${ }^{2}$ M. Krech and S. Dietrich, Phys. Rev. Lett. 66, 345 (1991).

${ }^{3}$ M. Krech and S. Dietrich, Phys. Rev. A 46, 1922 (1992).

${ }^{4}$ M. Krech, The Casimir Effect in Critical Systems (World Scientific, 1994).

${ }^{5}$ H. B. G. Casimir, Kon. Ned. Akad. Wet. Proc. 51, 793 (1948).

${ }^{6}$ A. Hanke, F. Schlesener, E. Eisenriegler, and S. Dietrich, Phys. Rev. Lett. 81, 1885 (1998).

${ }^{7}$ O. Vasilyev, A. Gambassi, A. Maciołek, and S. Dietrich, Phys. Rev. E 79, 041142 (2009).

${ }^{8}$ B. Derjaguin, Kolloid-Z. 69, 155 (1934).

${ }^{9}$ C. Hertlein, L. Helden, A. Gambassi, S. Dietrich, and C. Bechinger, Nature 451, 172 (2008)

${ }^{10}$ D. Beysens and D. Estève, Phys. Rev. Lett. 54, 2123 (1985).

${ }^{11}$ H. Guo, T. Narayanan, M. Sztuchi, P. Schall, and G. H. Wegdam, Phys. Rev. Lett. 100, 188303 (2008).

${ }^{12}$ D. Bonn, J. Otwinowski, S. Sacanna, H. Guo, G. Wegdam, and P. Schall, Phys. Rev. Lett. 103, 156101 (2009).

${ }^{13}$ V. D. Nguyen, S. Faber, Z. Hu, G. H. Wegdam, and P. Schall, Nat. Commun. 4, 1584 (2013).

${ }^{14}$ T. F. Mohry, A. Maciołek, and S. Dietrich, J. Chem. Phys. 136, 224902 (2012).

${ }^{15}$ T. F. Mohry, A. Maciołek, and S. Dietrich, J. Chem. Phys. 136, 224903 (2012).

${ }^{16}$ T. F. Mohry, S. Kondrat, A. Maciołek, and S. Dietrich, Soft Matter 10, 5510 (2014).

${ }^{17}$ V. D. Nguyen, M. T. Dang, T. A. Nguyen, and P. Schall, J. Phys.: Condens. Matter 28, 043001 (2016)

${ }^{18}$ M. T. Dang, A. V. Verde, P. G. Bolhuis, P. Schall et al., J. Chem. Phys. 139, 094903 (2013).

${ }^{19}$ J. R. Edison, N. Tasios, S. Belli, R. Evans, R. van Roij, and M. Dijkstra, Phys. Rev. Lett. 114, 038301 (2015).

${ }^{20}$ J. R. Edison, S. Belli, R. Evans, R. van Roij, and M. Dijkstra, Mol. Phys. 113, 2546 (2015).

${ }^{21}$ N. Tasios, J. R. Edison, R. van Roij, R. Evans, and M. Dijkstra, J. Chem. Phys. 145, 084902 (2016).

${ }^{22}$ J. R. Heringa and H. W. J. Blöte, Phys. Rev. E 57, 4976 (1998).

${ }^{23}$ J. Heringa and H. Blöte, Physica A 254, 156 (1998).

${ }^{24}$ D. J. Ashton, J. Liu, E. Luijten, and N. B. Wilding, J. Chem. Phys. 133, 194102 (2010).

${ }^{25} \mathrm{H}$. Hobrecht and A. Hucht, Phys. Rev. E 92, 042315 (2015).

${ }^{26}$ A. L. Talapov and H. W. J. Blöte, J. Phys. A: Math. Gen. 29, 5727 (1996).

${ }^{27}$ D. J. Ashton and N. B. Wilding, Mol. Phys. 109, 999 (2011).

${ }^{28}$ D. Ashton, N. Wilding, R. Roth, and R. Evans, Phys. Rev. E 84, 061136 (2011).

${ }^{29}$ M. E. J. Newman and G. T. Barkema, Monte Carlo Methods in Statistical Physics (Clarendon Press, Oxford University Press, New York, 1999).

${ }^{30}$ K. A. Maerzke, L. Gai, P. T. Cummings, and C. McCabe, J. Phys.: Conf. Ser. 487, 012002 (2014).

${ }^{31}$ F. Del Rio, E. Avalos, R. Espindola, L. F. Rull, G. Jackson, and S. Lago, Mol. Phys. 100, 2531 (2002).

${ }^{32}$ L. Vega, E. de Miguel, L. F. Rull, G. Jackson, and I. A. McLure, J. Chem. Phys. 96, 2296 (1992).

${ }^{33}$ D. L. Pagan and J. D. Gunton, J. Chem. Phys. 122, 184515 (2005).

${ }^{34}$ R. Guida and J. Zinn-Justin, J. Phys. A: Math. Gen. 31, 8103 (1998).

${ }^{35}$ A. Fortini and M. Dijkstra, J. Phys.: Condens. Matter 18, L371 (2006). 